\title{
A criterion for uniqueness in $G$-measures and perfect sampling
}

\author{
BY ANTHONY H. DOOLEY \\ School of Mathematics, University of New South Wales, Sydney 2052 Australia. \\ e-mail: a.dooley@unsw.edu.au \\ AND ÖRJAN STENFLO† \\ Department of Mathematics, Stockholm University, SE-10691 Stockholm, Sweden. \\ e-mail: stenflo@math.su.se
}

(Received 29 November 2004)

\section{Abstract}

Using coupling techniques, we prove uniqueness in $G$-measures under a weak regularity condition and give estimates of the associated rates of convergence. We also show how to generate a random variable distributed according to the unique $G$-measure on cylinder sets for any fixed level of precision.

\section{Introduction}

Let $\left\{X_{n}\right\}_{n \leqslant 0}$ be a stochastic process on $\{1, \ldots, N\}$. We may define random variables

$$
G_{k}(y):=P\left(X_{n}=y_{n}, 0 \geqslant n \geqslant-k+1 \mid X_{m}=y_{m}, m \leqslant-k\right),
$$

where $y=y_{0} y_{-1} y_{-2} \cdots$ and $k \geqslant 1$. Also, a.s.,

$$
G_{k}(y)=\prod_{i=0}^{k-1} g_{i}\left(\theta^{i} y\right),
$$

where $\theta$ denotes the shift map, and

$$
g_{i}(y)=P\left(X_{-i}=y_{0} \mid X_{-i+m}=y_{m}, m \leqslant-1\right) .
$$

This presents the measure defining $\left\{X_{n}\right\}_{n \leqslant 0}$ as a $G$-measure. If the set of functions $G=\left\{g_{i}\right\}_{i \geqslant 0}$, given by $(1 \cdot 1)$ uniquely specify this measure, then we say that there is a unique $G$-measure.

The concept of $G$-measures originates from Brown and Dooley [2] and is a generalisation of the notion of $g$-measure introduced by Keane [8]. Keane's work was based on the consideration of a $g$-measure as a shift invariant measure on an infinite product space, corresponding to the case when $\left\{X_{n}\right\}$ is stationary. (Note that $g=g_{i}$ is independent of $i$ if $\left\{X_{n}\right\}$ is stationary.)

One of the key questions asked in Keane's paper is whether continuity and positivity of $g$ was a sufficient condition for uniqueness, but this was disproved by Bramson and Kalikow [3], and by Quas [10] for circle continuous $g$-functions. 
The question of sufficient conditions for uniqueness in $g$-measures has a long history within the theory of "chains with complete connections" in the case $\left\{X_{n}\right\}$ is assumed to be stationary, with Doeblin and Fortet [5] and Harris [7] containing some of the most important early results.

The notion of $g$-measure corresponds to the idea of an equilibrium state in statistical mechanics in the special case of a normalized potential. It is not known whether the well known conditions of Hölder-continuity and "summable variations" for uniqueness in equilibrium-states for general potentials, see Bowen [1] and Walters [13] respectively, can be relaxed to the corresponding best known conditions for non-normalized potentials.

In recent work, Dooley and Hamachi [6] showed that every non-singular ergodic dynamical system is orbit equivalent to a Markov odometer with a unique $G$-measure. Therefore it is of heightened interest to give the best possible conditions for uniqueness.

Keane proved uniqueness in $g$-measures for strictly positive differentiable $g$ functions, unaware of the already existing weaker conditions for uniqueness by Doeblin and Fortet [5] of summable variations and the even weaker condition by Harris [7]. In Brown and Dooley [2], sufficient conditions were given for uniqueness in $G$-measures, generalising those of Keane. As with Keane's conditions, it is clear that these conditions are not necessary.

In this paper, we shall generalise the coupling ideas of Harris [7] for proving uniqueness in $g$-measures to the case of $G$-measures, showing how the coupling method work in this more general case. In the next section, we give the definitions of our basic notions, and a precise statement of the results. The basic theorem (Theorem 1 ) is proved in Section 3. As a consequence of our method of proving Theorem 1, we are able to give a perfect sampling algorithm in Theorem 2 .

\section{Preliminaries and statements of the results}

Let $\{N(j)\}_{j=0}^{\infty}$ be a sequence of positive integers, and let $\Sigma_{n}:=\prod_{j=n}^{\infty}\{1,2, \ldots, N(j)\}$ be a sequence of spaces. For each $n$, introduce a topology on $\Sigma_{n}$ by the metric

$$
\rho(x, y):= \begin{cases}2^{-j}, & \text { if } x \text { and } y \text { differ for the first time in the } \\ 0, & \text { if } x=y\end{cases}
$$

The spaces $\left(\Sigma_{n}, \rho\right)$ are compact metric spaces.

For $j \in\{1,2, \ldots, N(n)\}$ and $x=x_{1} x_{2} \cdots \in \Sigma_{n+1}$, let $j x$ be the element in $\Sigma_{n}$ defined by $j x=j x_{1} x_{2} \cdots$. Consider a family $\left\{g_{n}\right\}_{n=0}^{\infty}, g_{n}: \Sigma_{n} \rightarrow(0, \infty)$, of continuous functions, and suppose that the $g_{n}$ 's are normalised in the sense that

$$
\sum_{j=1}^{N(n)} g_{n}(j x)=1, \text { for any } x \in \Sigma_{n+1} .
$$

We call such a family $G:=\left\{g_{n}\right\}$ a family of $g$-functions.

Let $\Gamma_{n}$ denote the set of sequences $\gamma_{0} \gamma_{1} \cdots \gamma_{n-1}$ such that $\gamma_{j} \in\{1,2, \ldots, N(j)\}$, for any $0 \leqslant j \leqslant n-1$. For $\gamma=\gamma_{0} \cdots \gamma_{n-1} \in \Gamma_{n}$ and $x=x_{0} x_{-1} \cdots$ in $\Sigma_{0}$, let $\gamma(x)=\gamma_{0} \cdots \gamma_{n-1} x_{-n} x_{-(n+1)} \cdots$. 
Definition 1. Let $\mu^{n}$ denote the measure $\sum_{\gamma \in \Gamma_{n}} \mu \circ \gamma$, and let $G_{n}(x)=\prod_{i=0}^{n-1} g_{i}\left(\theta^{i} x\right)$, where $\theta$ denotes the shift map.

We say that a probability measure $\mu$ on $\Sigma_{0}$ is a $G$-measure if for any $n \geqslant 1$,

$$
\frac{d \mu}{d \mu^{n}}(x)=G_{n}(x)
$$

for $\mu$-almost all $x \in \Sigma_{0}$.

In Brown and Dooley [2] it was shown that, provided the functions $G_{i}$ are continuous, the existence of a unique $G$-measure is equivalent to the convergence (everywhere, or uniformly) of the sequence of functions

$$
\lim _{n \rightarrow \infty} \sum_{\gamma \in \Gamma_{n}} f(\gamma(x)) G_{n}(\gamma(x))
$$

for all $f \in C\left(\Sigma_{0}\right)$ and $x \in \Sigma_{0}$.

If $(2 \cdot 4)$ holds, then the limit is equal to $\int f d \mu$ for the unique $G$-measure. In Brown and Dooley [2] it was further shown that a unique $G$-measure is necessarily ergodic for the finite coordinate change action on $\Sigma_{0}$.

In the case when $N(j)$ is constant (say $=N$ for all $j$ ), we may identify $\Sigma_{n}$ with $\Sigma_{0}$ via the shift map. If $\mu$ is shift invariant i.e. $\mu \circ \theta=\mu$ then all the functions $g_{i}$ are identical under this identification, to a single function $g$, and we say that we have a $g$-measure. We are also interested in the case when there is a unique $g$-measure.

Notice that, in this setting, if there is a unique $G$-measure, there is necessarily a unique $g$-measure, since by definition if $\mu$ is a $g$-measure then $\mu$ is also a $G$-measure with $g_{i}=g$ for all $i$. However, the converse is not true. This is shown by the following.

Example 1. Let $N(j)=2$ for all $j$ and define

$$
g(x)=1, \text { if } x=01 * \text { or } x=10 *
$$

and $g(x)=0$ otherwise, where $*$ denotes an arbitrary ending of the infinite sequence $x$.

Let $x_{0}=010101 \ldots$ and $x_{1}=10101 \ldots$ It is not hard to see that the two Dirac measures $\delta_{x_{0}}$ and $\delta_{x_{1}}$ are both $G$-measures (associated with $g$ ), as is any convex combination of them. Thus we do not have uniqueness in $G$-measures. However, there is a unique $g$-measure (shift-invariant $G$-measure), viz. $(1 / 2)\left(\delta_{x_{0}}+\delta_{x_{1}}\right)$.

In this paper we show that there is a unique $G$-measure provided that

$$
\sum_{n=1}^{\infty} \prod_{m=1}^{n} c f f_{G}\left(2^{-m}\right)=\infty
$$

where

$$
c f f_{G}\left(2^{-m}\right)=\inf _{n} \inf _{1 \leqslant j_{l} \leqslant N(n+l), 1 \leqslant l \leqslant m-1} \sum_{i=1}^{N(n)} \inf _{y} g_{n}\left(i j_{1} \cdots j_{m-1} y\right) .
$$

The condition corresponding to $(2 \cdot 5)$ for uniqueness in $g$-measures was first considered by Comets et al. [4]. This condition is slightly stronger than the weakest known condition for uniqueness in $g$-measures of this type, see Stenflo [12], but has the advantage that it also gives the "uniform" convergence needed in our case. 
Theorem 1. Let $G$ be a family of $g$-functions satisfying condition $(2 \cdot 5)$.

Let $P$ denote the product Lebesgue measure on $\left((0,1)^{\mathbb{N}}, \mathcal{C}\right)$, where $\mathcal{C}$ is the product Borel $\sigma$-field on $(0,1)^{\mathbb{N}}$.

Then, for any $x \in \Sigma_{0}$, we can construct a sequence of random variables $\left\{\hat{Z}_{n}(x)\right\}$, $\hat{Z}_{n}(x):(0,1)^{\mathbb{N}} \rightarrow \Sigma_{0}$ with $P\left(\hat{Z}_{n}(x)=\gamma(x)\right)=G_{n}(\gamma(x))$, such that $\hat{Z}_{n}(x) \rightarrow \hat{Z}, P$ a.s., where $\hat{Z}:(0,1)^{\mathbb{N}} \rightarrow \Sigma_{0}$, is a random variable (independent of $x$ ). We have

$$
E \sup _{x \in \Sigma_{0}} \rho\left(\hat{Z}_{n}(x), \hat{Z}\right) \leqslant E 2^{-Y_{n}}
$$

where the metric $\rho$ is defined in (2.1) above, and $\left\{Y_{n}\right\}$ is a Markov chain with state space $\mathbb{N}$ starting at $Y_{0}=1$, with $P\left(Y_{n+1}=k+1 \mid Y_{n}=k\right)=c f f_{G}\left(2^{-k}\right)$, and $P\left(Y_{n+1}=1 \mid Y_{n}=k\right)=1-\operatorname{cff} f_{G}\left(2^{-k}\right)$, for any $k \geqslant 1$.

An explicit definition of $\hat{Z}_{n}(x)\left(=\hat{Z}_{n}(x, \omega)\right)$ is given in the proof below. The random variables $\hat{Z}_{n}(x, \omega)$ only depend on the first $n$ coordinates of $\omega \in(0,1)^{\mathbb{N}}$.

Remark 1. Note that $\left\{Y_{n}\right\}_{n=0}^{\infty}$ is a non-ergodic Markov chain under condition $(2 \cdot 5)$, see e.g. Prabhu [9, p. 80, example 18], so $E 2^{-Y_{n}} \rightarrow 0$.

Define $\mu(\cdot)=P(\hat{Z} \in \cdot)$. As a consequence of Theorem 1 and the well known fact that almost sure convergence implies convergence in distribution, see e.g. Shiryaev [11], we obtain.

Corollary 1. Let $G$ be a family of $g$-functions satisfying condition $(2 \cdot 5)$. Then there exists a unique $G$-measure, $\mu$, i.e.

$$
\lim _{n \rightarrow \infty} \sum_{\gamma \in \Gamma_{n}} f(\gamma(x)) G_{n}(\gamma(x))=\int f d \mu
$$

for all $f \in C\left(\Sigma_{0}\right)$ and $x \in \Sigma_{0}$.

Even though the definition of $\mu$ is implicit, we can correctly simulate $\mu$-distributed random variables up to any specified degree of accuracy. The following theorem generalizes results from Comets et al. [4].

Theorem 2 (Perfect sampling). Let $G$ be a family of $g$-functions satisfying condition $(2 \cdot 5)$.

For $s \in(0,1)$, and integers $m \geqslant 1$, let $f_{s}(m)=m+1$, if $s<c f f_{G}\left(2^{-m}\right), f_{s}(m)=1$ if $s \geqslant \operatorname{cff} f_{G}\left(2^{-m}\right)$.

Let $N_{\star}$ be an arbitrary fixed integer.

Algorithm: generate independent, uniformly distributed random variables on the unit interval, $U_{1}, U_{2}, \ldots, U_{T}$, where the stopping time $T$ is the first integer such that $f_{U_{1}} \circ$ $\cdots \circ f_{U_{T}}(1)>N_{\star}$.

Let $\mu$ be the unique G-measure. Then the first $N_{\star}$ (common) coordinates of $\hat{Z}_{N_{\star}}(x)$ (defined in the proof below) is a random variable taking value $\left(i_{0}, \ldots, i_{N_{\star}-1}\right)$ with probability $\mu\left(\left[i_{0}, \ldots, i_{N_{\star}-1}\right]\right)$, for an arbitrary cylinder set $\left[i_{0}, \ldots, i_{N_{\star}-1}\right]=\left\{x \in \Sigma_{0}: x_{j}=i_{j}\right.$, $\left.0 \leqslant j \leqslant N_{\star}-1\right\}$, of length $N_{\star}$ in $\Sigma_{0}$.

\section{Proofs}

Fix an integer $n \geqslant 0$, and $\omega \in(0,1)^{\mathbb{N}}$. We first define the function $\hat{Z}_{n}: \Sigma_{0} \times(0,1)^{\mathbb{N}} \rightarrow$ $\Sigma_{0}$. 
Intuitively, for $y=y_{0} y_{-1} y_{-2} \cdots \in \Sigma_{0}, \hat{Z}_{n}(y, \omega)$ will correspond to the "history available" at time 0 of a realization $\omega$ of a stochastic sequence with conditional distributions prescribed by the family of $g$-functions $G$, if the "history available" at time $-n$ is fixed to be $y_{-n} y_{-n+1} \cdots$.

To make this intuition precise, let for $\omega=\omega_{0} \omega_{1} \omega_{2} \cdots \in(0,1)^{\mathbb{N}}$, and $y \in \Sigma_{0}$,

$$
\hat{Z}_{n}(y, \omega):=X_{0}^{n}\left(\theta^{n} y, \omega\right),
$$

where $\left\{X_{j}^{n}(x, \omega)\right\}_{j=-n}^{0}$ is a sequence of functions $X_{-j}^{n}: \Sigma_{n} \times(0,1)^{\mathbb{N}} \rightarrow \Sigma_{j}$ defined recursively in the following way.

Let $X_{-n}^{n}(x, \omega)=x$, for any $x \in \Sigma_{n}$. Suppose that for some $k_{0}, X_{-\left(k_{0}+1\right)}^{n}(x, \omega)$ has already been defined. We then proceed to define $X_{-k_{0}}^{n}(x, \omega)$ as follows. Let $M=M(\omega)$ be the largest integer such that $X_{-\left(k_{0}+1\right)}^{n}(x, \omega)$ belongs to the cylinder set $\left\{i_{1} i_{2} \cdots i_{M} y\right.$ : $\left.y \in \Sigma_{k_{0}+1+M}\right\}$, for some $i_{j} \in\left\{1, \ldots, N\left(k_{0}+j\right)\right\}, j=1, \ldots, M$, and any $x \in \Sigma_{n}$.

For $1 \leqslant j \leqslant N\left(k_{0}\right)$, let

$$
\begin{gathered}
A_{0}(j):=\left\{s \in(0,1): \sum_{i=1}^{j-1} \inf _{y} g_{k_{0}}(i y) \leqslant s<\sum_{i=1}^{j} \inf _{y} g_{k_{0}}(i y)\right\} \\
A_{1}\left(j i_{1}\right):=\left\{s \in(0,1): \sum_{i=1}^{N\left(k_{0}\right)} \inf _{y} g_{k_{0}}(i y)+\sum_{i=1}^{j-1}\left(\inf _{y} g_{k_{0}}\left(i i_{1} y\right)-\inf _{y} g_{k_{0}}(i y)\right)\right. \\
\left.\leqslant s<\sum_{i=1}^{N\left(k_{0}\right)} \inf _{y} g_{k_{0}}(i y)+\sum_{i=1}^{j}\left(\inf _{y} g_{k_{0}}\left(i i_{1} y\right)-\inf _{y} g_{k_{0}}(i y)\right)\right\}
\end{gathered}
$$

and for $m \geqslant 2$,

$$
\begin{aligned}
A_{m}\left(j i_{1} \cdots i_{m}\right):= & \left\{s \in(0,1): \sum_{i=1}^{N\left(k_{0}\right)} \inf _{y} g_{k_{0}}\left(i i_{1} \cdots i_{m-1} y\right)\right. \\
& +\sum_{i=1}^{j-1}\left(\inf _{y} g_{k_{0}}\left(i i_{1} \cdots i_{m} y\right)-\inf _{y} g_{k_{0}}\left(i i_{1} \cdots i_{m-1} y\right)\right) \\
\leqslant & s<\sum_{i=1}^{N\left(k_{0}\right)} \inf _{y} g_{k_{0}}\left(i i_{1} \cdots i_{m-1} y\right) \\
& \left.+\sum_{i=1}^{j}\left(\inf _{y} g_{k_{0}}\left(i i_{1} \cdots i_{m} y\right)-\inf _{y} g_{k_{0}}\left(i i_{1} \cdots i_{m-1} y\right)\right)\right\} .
\end{aligned}
$$

Define $X_{-k_{0}}^{n}(x, \omega)=j X_{-\left(k_{0}+1\right)}^{n}(x, \omega)$, if $\omega_{k_{0}} \in \bigcup_{k=0}^{M} A_{k}\left(j i_{1} \cdots i_{k}\right)$, or

$$
\begin{aligned}
\omega_{k_{0}} \in\{ & s \in(0,1): \sum_{i=1}^{N\left(k_{0}\right)} \inf _{y} g_{k_{0}}\left(i i_{1} \cdots i_{M} y\right) \\
& +\sum_{i=1}^{j-1}\left(g_{k_{0}}\left(i X_{-\left(k_{0}+1\right)}^{n}(x, \omega)\right)-\inf _{y} g_{k_{0}}\left(i i_{1} \cdots i_{M} y\right)\right) \\
& \leqslant s<\sum_{i=1}^{N\left(k_{0}\right)} \inf _{y} g_{k_{0}}\left(i i_{1} \cdots i_{M} y\right) \\
& \left.+\sum_{i=1}^{j}\left(g_{k_{0}}\left(i X_{-\left(k_{0}+1\right)}^{n}(x, \omega)\right)-\inf _{y} g_{k_{0}}\left(i i_{1} \cdots i_{M} y\right)\right)\right\} .
\end{aligned}
$$


Let $P$ be the product Lebesgue measure on $(0,1)^{\mathbb{N}}$. By construction $P\left(X_{-k_{0}}^{n}(x, \omega)=j \gamma_{k_{0}+1} \cdots \gamma_{n-1} x \mid X_{-\left(k_{0}+1\right)}^{n}(x, \omega)=\gamma_{k_{0}+1} \cdots \gamma_{n-1} x\right)=g_{k_{0}}\left(j \gamma_{k_{0}+1} \cdots \gamma_{n-1} x\right)$.

Thus $X_{-k}^{n}(x, \omega)$ can be viewed as random variables, for each fixed $x$, with

$$
P\left(X_{-k}^{n}(x, \omega)=\gamma_{k} \cdots \gamma_{n-1} x\right)=\prod_{i=k}^{n-1} g_{i}\left(\gamma_{i} \cdots \gamma_{n-1} x\right)
$$

(In the formula above we have calculated the probability on the left as a product of a finite collection of conditional probabilities.)

Recall the definition of the metric $\rho$ in $(2 \cdot 1)$. Define the random variables $D_{j}^{n}(\omega):=$ $\sup _{x, y} \rho\left(X_{j}^{n}(x, \omega), X_{j}^{n}(y, \omega)\right)$. Then by construction, from $(3 \cdot 1)$,

$$
\sup _{x, y \in \Sigma_{0}} \rho\left(\hat{Z}_{n}(x, \omega), \hat{Z}_{n}(y, \omega)\right) \leqslant D_{0}^{n}(\omega)
$$

and

$$
\begin{aligned}
P\left(D_{-k_{0}}^{n}=2^{-(M+2)} \mid D_{-\left(k_{0}+1\right)}^{n}=2^{-(M+1)}\right) & \\
& \geqslant \inf _{n} \inf _{1 \leqslant j_{l} \leqslant N(n+l), 1 \leqslant l \leqslant M} \sum_{i=1}^{N(n)} \inf _{y} g_{n}\left(i j_{1} \cdots j_{M} y\right)=c f f_{G}\left(2^{-(M+1)}\right),
\end{aligned}
$$

for any $0 \leqslant k_{0} \leqslant n-1, n \geqslant 1$.

Define

$$
\hat{Y}_{n}(\omega)=f_{\omega_{0}} \circ \cdots \circ f_{\omega_{n-1}}(1), n \geqslant 1 \quad \hat{Y}_{0}(\omega)=1,
$$

where for $s \in(0,1)$, and integers $m \geqslant 1, f_{s}(m)=m+1$, if $s<c f f_{G}\left(2^{-m}\right), f_{s}(m)=1$ if $s \geqslant c f f_{G}\left(2^{-m}\right)$. Then $\hat{Y}_{n}(\omega)$ is nondecreasing in $n$, and since $D_{-(n-k)}^{n}(\omega) \leqslant 2^{-\hat{Y}_{k}(\omega)}$, for any $0 \leqslant k \leqslant n$, we obtain in the particular case (when $k=n$ ) using $(3 \cdot 2)$ that

$$
\sup _{x, y \in \Sigma_{0}} \rho\left(\hat{Z}_{n}(x, \omega), \hat{Z}_{n}(y, \omega)\right) \leqslant 2^{-\hat{Y}_{n}(\omega)}
$$

for any $\omega \in(0,1)^{\mathbb{N}}$. In particular this means that if $\hat{Y}_{T}(\omega)>N_{\star}$, for some $T=T(\omega)$, then the first $N_{\star}$ digits of $\hat{Z}_{n}(x, \omega)$ do not depend on $x$ for any $n \geqslant T(\omega)$. Thus the proof of Theorem 2 will be completed if we prove that $\hat{Y}_{n} \rightarrow \infty$ as $n \rightarrow \infty$ a.s.

Let $\left\{Y_{n}\right\}_{n=0}^{\infty}$, be a stochastic sequence with $Y_{n}:(0,1)^{\mathbb{N}} \rightarrow \mathbb{N}, n \geqslant 0$, defined inductively in the following way: let $Y_{0}(\omega)=1$, for all $\omega \in(0,1)^{\mathbb{N}}$. Suppose $Y_{n}(\omega)=m$. Let $Y_{n+1}(\omega)=m+1$ if $\omega_{n+1}<c f f_{G}\left(2^{-m}\right)$ and $Y_{n+1}(\omega)=1$ otherwise.

It follows that $\left\{Y_{n}\right\}$ is a homogeneous Markov chain with $Y_{0}=1$,

$$
P\left(Y_{n+1}=m+1 \mid Y_{n}=m\right)=c f f_{G}\left(2^{-m}\right)
$$

and

$$
P\left(Y_{n+1}=1 \mid Y_{n}=m\right)=1-c f f_{G}\left(2^{-m}\right), m \geqslant 1 .
$$

Note that $Y_{n}$ and $\hat{Y}_{n}$ are identically distributed for any fixed $n$. Therefore, by $(3 \cdot 3)$,

$$
E \sup _{x, y \in \Sigma_{0}} \rho\left(\hat{Z}_{n}(x), \hat{Z}_{n}(y)\right) \leqslant E 2^{-Y_{n}}, n \geqslant 0 .
$$

Since $Y_{n}$ is a non-ergodic Markov chain by assumption, see e.g. Prabhu [9, p. 80, example 18], and $\hat{Y}_{n}$ is monotone, it follows that $\hat{Y}_{n} \rightarrow \infty$ a.s. as $n \rightarrow \infty$, and thus 
using $(3 \cdot 3)$, we obtain

$$
\sup _{x, y \in \Sigma_{0}} \rho\left(\hat{Z}_{n}(x), \hat{Z}_{n}(y)\right) \rightarrow 0, \text { a.s. }
$$

as $n \rightarrow \infty$.

Note that if for some $M \leqslant n-k_{0}, \omega_{k_{0}+j} \in \bigcup_{k=0}^{M-j} A_{k}\left(i_{j+1} \cdots i_{j+k}\right)$ for all $j=0, \ldots$, $M-1$, then $X_{-k_{0}}^{n} \in\left\{i_{1} i_{2} \cdots i_{M} y: y \in \Sigma_{k_{0}+M}\right\}$ for all $n \geqslant M$.

From this property (in the case $k_{0}=0$ ) in combination with $(3 \cdot 5)$ it follows that there exists a $\Sigma_{0}$-valued random variable $\hat{Z}$, such that $\hat{Z}_{n}(x, \omega)$ converges almost surely to $\hat{Z}(\omega)$, uniformly in $x$. From $(3 \cdot 4)$ it follows that

$$
E \sup _{x \in \Sigma_{0}} \rho\left(\hat{Z}_{n}(x), \hat{Z}\right) \leqslant E 2^{-Y_{n}}
$$

This completes the proofs of the theorems.

\section{REFERENCES}

[1] R. Bowen. Equilibrium States and the Ergodic Theory of Anosov Diffeomorphisms. Lecture Notes in Mathematics, vol. 470 (Springer-Verlag, 1975).

[2] G. Brown and A. H. Dooley. Odometer actions on G-measures. Ergod. Th. Dynam. Sys. 11 (1991), 279-307.

[3] M. Bramson and S. Kalikow. Non-uniqueness in $g$-functions. Israel J. Math. 84 (1993), 153160.

[4] F. Comets, R. Fernández and P. A. Ferrari. Processes with long memory: regenerative construction and perfect simulation. Ann. Appl. Probab. 12 (2002), 921-943.

[5] W. Doeblin and R. Fortet. Sur les chaines à liaisons complètes. Bull. Soc. Math. France 65 (1937), 132-148.

[6] A. H. Dooley and T. Hamachi. Nonsingular dynamical systems, Bratteli diagrams and Markov odometers. Israel J. Math. 138 (2003), 93-123.

[7] T. E. Harris. On chains of infinite order. Pacific J. Math. 5 (1955), 707-724.

[8] M. Keane. Strongly mixing g-measures. Invent. Math. 16 (1972), 309-324.

[9] N. U. Prabhu. Stochastic Processes. Basic Theory and its Applications (Macmillan, 1965).

[10] A. QUAS. Non-ergodicity for $C^{1}$-expanding maps and $g$-measures. Ergod. Th. Dynam. Systems 16 (1996), 531-543.

[11] A. N. Shir Yaev. Probability, 2nd ed. (Springer, 1996).

[12] Ö. Stenflo. Uniqueness in $g$-measures. Nonlinearity 16 (2003), 403-410.

[13] P. Walters. Ruelle's operator theorem and g-measures. Trans. Amer. Math. Soc. 214 (1975), $375-387$. 